\title{
Représentations sociales de la contention en Pédiatrie : regards de professionnels
}

\author{
Titre court : Représentations sociales de la contention
}

Social representations of contention in Pediatric: Looks of professionals

\section{Social representations of contention}

C. Oudjani a, L. Dany a,b, ${ }^{*}$, M. Derome c, J. Bataille c

a Aix-Marseille Université, LPS EA 849, 13621, Aix-en-Provence, France

b APHM, Timone, Service d'Oncologie Médicale, 13385, Marseille, France

${ }^{\mathrm{c}}$ Hôpital Raymond Poincaré, Pôle de Pédiatrie - Réanimation Infantile - Rééducation Neurorespiratoire, 92380, Garches, France

Auteur correspondant :

Lionel Dany

Laboratoire de psychologie sociale

Université d'Aix-Marseille

Maison de la Recherche

29, avenue Robert-Schuman

13621 Aix-en-Provence cedex 1, France.

Mail : Lionel.Dany@univ-amu.fr

Tel : ++33 442.953.819. 


\title{
Résumé
}

Objectifs. Cette étude s'inscrit dans un contexte de remise en question actuelle de la pratique de la contention dans le champ du soin au niveau national et international. Elle questionne la façon dont les représentations sociales de la contention organisent et façonnent le discours des professionnels dans un contexte de soins et de prise en charge de l'enfant malade et/ou handicapé. L'objectif principal était de comprendre comment ces représentations sociales intervenaient dans la pratique de la contention.

Population et méthodes. La méthode qualitative, à travers des entretiens semi-directifs, a été choisie pour tenter de répondre à nos objectifs. La recherche s'est déroulée auprès de 15 professionnels du service de Pédiatrie - Réanimation Infantile - Rééducation neurorespiratoire de l'Hôpital Raymond Poincaré. Les données ont été traitées à partir d'une analyse de contenu de type thématique.

Résultats. Les résultats laissent à penser que les représentations sociales affectent en particulier le vécu des professionnels et la représentation qu'ils peuvent avoir du vécu des enfants, des parents et du rôle de ces derniers dans la contention. Ils montrent également que la contention est un objet pluridimensionnel dont les contours sont difficiles à appréhender, notamment en raison du contexte actuel de remise en question de cette pratique au sein du service.

Conclusion. Malgré certaines limites méthodologiques, cette étude a contribué à une démarche réflexive autour de la pratique de la contention, dans une approche psychosociale de compréhension de l'objet et de ses enjeux.

Mots clés : contention ; représentations sociales ; enfants malades et/ou handicapés

\begin{abstract}
Summary
Aims. This study is part of a context of current questioning practice of restraint in the field of care at national and international level. It asks how social representations of restraint organize and shape the discourse in the context of professional care and support for sick and/or disabled children. The main objective was to understand how these social representations were involved in the practice of restraint.
\end{abstract}

Population and Methods. Qualitative method, through semi-structured interviews was chosen to try to meet our goals. The research was conducted with 15 professional service Pediatrics - Infant Intensive Care - Neuro-Rehabilitation Respiratory of the Hospital Raymond Poincaré. The data were processed using a content analysis of the thematic type.

Results. The results suggest that social representations affect particularly experienced and professional representation they can have about the experience of children, parents and the role of the latter in restraint. They also show that restraint is a multidimensional object whose contours are difficult to understand, particularly because of the current context of questioning this practice within the service.

Conclusion. Despite some methodological limitations, this study has contributed to a reflective process around the practice of restraint in a psychosocial approach of understanding the subject and its issues.

Keywords : restraint ; social representations ; sick and/or disabled children 


\section{Introduction}

La contention est une pratique internationale ancienne et culturellement ancrée dans le champ du soin. Depuis quelques années, elle représente un objet de questionnement éthique national qui s'inscrit dans un contexte où la France serait un des pays européens les plus utilisateurs de contention [1]. Le fait qu'il s'agisse d'un processus de soins à risque [2] semble avoir contribué à ce questionnement. Certains auteurs mettent ainsi en avant les dangers de cette pratique, tels que les abus de contention [3], l'absence de preuves de l'efficacité thérapeutique et l'existence de conséquences néfastes pour la personne [1,3]. Ce questionnement se retrouve également au niveau international autour de la question de la régulation des pratiques suite à plusieurs décès observés après la mise en place d'une contention [4].

La définition de la contention est entourée d'un flou sémantique aussi bien au niveau national qu'international. Certains auteurs parlent de contention "chimique», "relationnelle», «physique» et "mécanique»[3]. La contention chimique, tout d'abord, consiste à administrer des sédatifs (neuroleptiques ou tranquillisants). La contention dite relationnelle consiste à «tenir» une personne par la parole. La contention physique correspond au maintien de la personne par certaines techniques thérapeutiques. Enfin, la contention dite mécanique renvoie à des mesures spécifiques (fixation de ceintures au lit, isolement). Cependant, la distinction entre ces deux derniers types n'est pas toujours bien établie dans la littérature. En ce qui concerne la contention physique, l'Agence Nationale d'Accréditation et d'Évaluation en Santé [2] distingue la contention physique dite active (à visée rééducative) de la contention physique dite passive (à visée sécuritaire). C'est à ce dernier type de contention que la plupart des études se sont intéressées. Différents moyens de contention peuvent être employés pour contenir une personne (sangles thoraciques, attaches de poignets et de cheville, barrières de lit, etc.). D'autres moyens dits «non spécifiques » peuvent également être mis en place, tels que l'utilisation d'un drap limitant les mouvements volontaires [2]. Principalement connue pour son utilisation en psychiatrie, la contention est également employée en gériatrie, dans les services adultes et de pédiatrie, en réanimation ou encore en orthopédie. La littérature, peu développée sur le sujet, ne met pas en avant de rôle ou de fonction spécifique de la contention dans le champ pédiatrique. Celle-ci répondrait aux mêmes besoins que lorsqu'elle est mise en place dans les autres domaines de soin. Son but est de prévenir une violence du patient envers lui-même ou autrui alors que les autres moyens de contrôle ne sont ni efficaces, ni appropriés ou de prévenir un risque de rupture thérapeutique [5].

La contention n'a de sens que si elle est pensée dans la relation à l'autre [6]. Plus précisément, elle est, par nature, un objet social car elle implique la présence d'un professionnel et d'un 
patient qui entretiennent tous deux un rapport à cet objet et qui se trouvent chacun dans un contexte social spécifique. Dans le cadre pédiatrique, la contention implique également un autre acteur important : les parents.

Dans ce contexte, il nous a paru pertinent de questionner la contention d'un point de vue psychosocial [7] en étudiant les représentations sociales de la contention auprès de soignants exerçant dans un service de Pédiatrie. Par représentations sociales, nous entendons l'ensemble organisé des connaissances, des croyances, des opinions, des images et des attitudes partagées par un groupe à l'égard d'un objet social donné [6]. Les représentations sociales ont différentes fonctions : une fonction savoir (elles permettent de comprendre et d'expliquer la réalité) ; une fonction de guide pour l'action (elles définissent la finalité des situations en lien avec l'objet, constituent des systèmes d'attentes/d'anticipations); une fonction identitaire (elles définissent l'identité du groupe et permettent la sauvegarde de sa spécificité), et une fonction justificatrice (elles permettent a posteriori de justifier les comportements et prises de position) [6].

Un certain nombre de travaux ont montré que l'étude des représentations sociales est des plus pertinentes dans le cadre du soin. En effet, l'interaction thérapeutique s'opère bien souvent autour et par les représentations que chacun des acteurs de cette situation élabore à propos de la maladie, du soin voire du rôle qu'il est en mesure de tenir dans la relation thérapeutique [810]. Les représentations sont constitutives de notre participation au monde social, de nos expériences à la fois individuelles et groupales. Par exemple, étudier les représentations sociales de la contention ne revient pas à mesurer l'écart entre les connaissances objectives voire les définitions institutionnelles de la contention et les savoirs produits par les professionnels. Il s'agit davantage de comprendre quelle(s) place(s) la contention occupe pour les professionnels et quels sens ce type d'intervention peut prendre dans le contexte d'une pratique professionnelle.

Notre question de recherche vise donc à étudier les représentations sociales de la contention auprès de professionnels de santé qui interviennent auprès d'enfants (malades et/ou handicapés) confrontés à des situations particulièrement invalidantes et nécessitant le recours à ces pratiques. L'objectif de ce travail est (1) d'étudier comment l'objet contention est reconstruit par les professionnels sans leur imposer un type de contention particulier, (2) d'appréhender la façon dont les représentations de la contention organisent et façonnent le discours des professionnels et (3) de mieux comprendre comment ces représentations interviennent dans l'explicitation et la mise en œuvre des pratiques de contention dans le cadre de la pédiatrie. 


\section{Population et méthodes}

\subsection{Lieu de l'étude}

Notre étude s'est déroulée auprès de professionnels du service de Pédiatrie - Réanimation Infantile - Rééducation neuro-respiratoire de l'Hôpital Raymond Poincaré. Ce service accueille des enfants lourdement handicapés à la suite d'accidents, de maladies congénitales, chroniques et/ou dégénératives. Le service est constitué de trois unités : l'unité de réanimation (10 lits), l'unité de surveillance continue (11 lits), l'unité de neuro-pédiatrie (14 lits), soit 35 lits au total. Elles permettent une prise en charge pluridisciplinaire, adaptée, spécialisée et ininterrompue des enfants, que ce soit au niveau du diagnostic, de la thérapeutique ou de la rééducation, ainsi que des bilans et du traitement de pathologies pédiatriques d'ordre général. Les enfants sont hospitalisés dans des chambres à deux ou trois lits, excepté en réanimation où ils sont placés en « boxe » individuel. La plupart des patients hospitalisés sont grandement « techniqués » (trachéotomie, halos, fauteuils roulants, etc.). Certains enfants sont hospitalisés en raison d'un risque vital immédiat (intoxication, noyade, etc.). Ainsi, la mise en place de la contention s'effectue dans un contexte où les enfants pris en charge sont lourdement « techniqués » et handicapés dans la majeure partie des cas, avec un risque vital pour certains d'entre eux.

\subsection{Procédure}

La méthode utilisée a été celle de l'entretien semi-directif. Ce type d'entretien permet que le sujet exprime sa réalité singulière, verbalisée au travers du langage [12]. Le guide d'entretien a été construit en deux temps. Une première version a été élaborée suite à une revue de la littérature disponible. Une fois les premiers entretiens réalisés avec cette version du guide, nous avons procédé à une analyse de contenu qui nous a permis de l'adapter au vu des données émergeant du terrain. Il était articulé autour de trois thèmes principaux : le travail dans le service, le travail sur ou avec le corps de l'enfant et la contention. Chacun de ces thèmes comportait une série de questions portant notamment sur la représentation et le vécu des soignants. Les participants devaient signer un formulaire de consentement éclairé et remplissaient une fiche signalétique qui nous permettait d'appréhender certaines caractéristiques de notre population (sexe, âge, profession et ancienneté). L'avis d'un comité d'éthique n'a pas été sollicité car le projet mis en oeuvre était en adéquation avec le code de conduite des chercheurs dans les sciences du comportement humain. Les entretiens se 
déroulaient soit dans le bureau des Psychologues du service, soit dans un bureau mis à disposition des professionnels.

\subsection{L'analyse de contenu}

Le matériel recueilli par l'intermédiaire des entretiens a été intégralement retranscrit et a fait l'objet d'une analyse de contenu, plus précisément une analyse thématique. L'analyse de contenu consiste en la mise ne œuvre de procédures systématiques et objectives de description du contenu des entretiens réalisés. Son but est l'inférence de connaissances relatives aux conditions de production (ou éventuellement de réception), à l'aide d'indicateurs (quantitatifs ou non) [13]. Dans cette recherche, le principal indicateur utilisé était la fréquence d'apparition des éléments. L'absence d'évocation de certaines caractéristiques était également prise en considération. L'analyse thématique consiste à repérer les unités sémantiques qui constituent l'univers discursif de l'énoncé [14]. Elle fonctionne par opérations de découpage du texte en unités de sens puis classification de ces unités en catégories selon des regroupements analogiques [13]. C'est à partir de ces catégories que le chercheur peut faire des inférences et des interprétations relatives à son objet d'étude. C'est autour des principales catégories qui ont été identifiées que s'articule la présentation de nos résultats.

\section{Résultats}

$\mathrm{Au}$ total, 15 professionnels de l'équipe pluridisciplinaire du service (médecin, interne, cadre de santé, auxiliaires de puériculture, aides-soignantes, infirmières, éducatrice de jeunes enfants, kinésithérapeutes, ergothérapeute, institutrice) ont participé à cette recherche. Les entretiens étaient d'une durée moyenne de 67,5 minutes. Le recueil des données s'est étendu de février à juin 2012. Les professionnels sont âgés de 25 à 52 ans (Moyenne = 36,5). L'échantillon était composé de 13 femmes et 2 hommes. La durée moyenne de travail dans le service était de 8 ans et 3 mois, allant de 3 mois à 28 ans. Les personnes incluses dans l'étude étaient celles qui avaient lu, compris et signé le formulaire de consentement éclairé.

L'analyse de contenu thématique a permis de faire émerger cinq thèmes principaux : (1) formes et causes de la contention; (2) processus décisionnels et mise en place de la contention ; (3) limites, cadre et alternatives à la contention ; (4) vécus de la contention et (5) le rapport au corps. Chacun de ces thèmes généraux contient un nombre plus ou moins important de sous-thèmes. Les fréquences d'évocation de ces sous-thèmes, leur articulation aux thèmes généraux et des extraits typiques d'entretiens sont présentées dans le tableau 1. 


\subsection{Formes et causes de la contention}

La forme de contention la plus évoquée par les professionnels était le fait «d'attacher» un enfant, les «attaches». Tous les professionnels ont évoqué cette thématique. Les autres formes de contention évoquées le plus fréquemment étaient les «barrières de lit», le fait de « maintenir » ou de «tenir » l'enfant, les «menottes », la « ceinture abdominale mise au lit», la « ceinture au fauteuil » et les «bas de contention ». L' " appareillage » (corset, attèles, etc.) a été cité par un quart des participants, dont la majorité étaient des professionnels de rééducation. La « contention chimique » n’a été évoquée que par une seule personne.

Concernant l'adaptation de la contention, trois formes principales ont été évoquées spontanément par les professionnels. Elles se rapportaient à l'adaptation à l'enfant, à la durée et l'évolution de la situation, et aux parents. Les causes de la mise en place de la contention les plus souvent évoquées par les professionnels étaient l'état du patient, des causes spécifiques à la Pédiatrie (e.g., «âge » de l'enfant), à la Réanimation et aux Soins Intensifs (enfant techniqué ou sédaté). D’autre part, près des trois quarts des professionnels ont mentionné le danger et le risque pour le patient. Ce qui est mis en avant c'est la «protection de l'enfant», le risque qu'il a «de se blesser», de «chuter» ou «d'arracher l'appareillage ». Le fait que ce soit «pour l'enfant», «pour son bien » a été exprimé par près des deux tiers des professionnels.

Pour près d'un soignant sur deux, la cause de la contention est qu'elle " permet le soin » ou qu'elle «fait partie du soin » et qu'il n'y a «pas d'autres choix ». La « peur » ou «l'inquiétude » des soignants sont évoqués par un tiers des professionnels. La protection et la sécurité des autres enfants et celle des soignants ont été également exprimées par les professionnels. L'expérience relative à des incidents antérieurs en lien avec la contention a été évoquée par un quart des personnes. Enfin, pour les deux tiers des participants, la contention était mise en place car elle est « systématique » et/ou « obligatoire ». Elle est «protocolisée », c'est une «évidence » pour la moitié des personnes interviewées.

\subsection{Processus décisionnels et mise en place de la contention}

Pour plus de la moitié des professionnels, la décision de la contention était prise par le médecin. En revanche, pour certains, elle serait davantage prise par les soignants. La décision prise par les parents ou avec eux a été évoquée par un professionnel su cinq. Enfin les demandes des enfants et des parents ont été évoquées chacune par une minorité (20\%) des participants. 
En ce qui concerne la mise en place de la contention, les résultats ont tout d'abord mis en évidence le fait que tous les professionnels interrogés ont déjà mis en place une contention. Un tiers des professionnels a expliqué que la mise en place se faisait avec l'accord des médecins et, plus rarement, sur prescription médicale. La fréquence d'utilisation a, quant à elle, été peu évoquée. Pour certain, elle est très utilisée pour d'autres elle est utilisée ponctuellement. Notons également que l'accord de l'enfant n'a été évoqué que par un professionnel sur dix.

En ce qui concerne la manière dont la contention devrait être mise en place, l'importance de la communication de manière générale, et avec l'enfant et les parents en particulier est mise en avant. Le fait d'avoir la possibilité d'en discuter entre professionnels a été exprimé par près des trois quarts des participants. De manière transversale, l'importance de la compréhension de la contention (enfant, parents et soignants) a été évoquée par les deux tiers $(67 \%)$ des participants. Le rôle des parents dans le processus décisionnel aussi bien que dans la mise en place de la contention a semblé assez flou pour une moitié des professionnels. Ce rôle «flou » des parents doit s'accompagner d'explication pour qu'elles soient transmises à l'enfant, de la participation active dans certains cas, de l'adhésion à la contention ou encore de l'apprentissage à la mise en place de la contention.

\subsection{Limites, cadre et alternatives}

Les limites de la contention ont été spontanément évoquées par près de la moitié des professionnels interrogés, en particulier les dangers de la contention et le risque de blessure et/ou de douleur. Relativement au cadre de cette pratique, pour près de la moitié des professionnels, la contention nécessitait une prescription médicale. Cet encadrement a été déclaré comme étant «nouveau » par un professionnels sur cinq et comme étant en évolution actuellement par une minorité. Certains ont déclaré ne pas savoir pourquoi la contention devait être mise en place sur prescription médicale. La question de la responsabilité du soignant a, quant à elle, été évoquée par une minorité de professionnels. La législation a, quant à elle, été évoquée par une seule personne. Les discussions et débat actuels autour de l'objet sont évoqués par un tiers des personnes.

Thème abordé spontanément par plus de la moitié des professionnels, les alternatives à la contention n'ont été évoquées ni par les médecins ni par les auxiliaires de puéricultures de l'échantillon. Les trois alternatives évoquées par les professionnels ont été la protection et/ou la mise en place de matériel, l'accompagnement de l'enfant et l'administration de médicaments. 


\subsection{Vécus de la contention}

Que ce soit pour les professionnels, les parents ou les enfants, le vécu négatif a été le plus fréquemment évoqué. Il concerne plus des deux tiers des interviewés.

Vécu des soignants - Des affects positifs ont été évoqués par près de la moitié des participants avec des termes tels que « contentement », « bien-être », « sentiment d'apaisement » ou d'être « rassuré ». En revanche, les deux tiers des professionnels ont évoqué des affects négatifs, la contention est « désagréable », "difficile », ça fait « mal au cour », c'est « dur », ça ne fait «pas plaisir», enfin, elle engendre de la «culpabilité ».

Vécu perçu des parents - Les affects négatifs ont été relatés par un tiers des participants qui estiment que pour les parents c'est « difficile », " dur », qu'ils " n'aiment pas », «n'apprécient pas », « vivent mal » la contention. Les affects positifs, peu évoqués, sont principalement associés à la fonction de réassurance de la contention.

Vécu perçu des enfants - Les affects négatifs associés aux vécus des enfants ont été évoqués par près des trois quarts des participants avec des expressions et termes tels que « mal vécu», « difficile », « douleur », « stress », « angoisse » ou « traumatisme ». Les affects positifs ont été évoqués par un quart des participants. Enfin, un professionnel sur dix a mentionné les affects négatifs éprouvés par les enfants lorsque la contention était retirée.

\subsection{Le rapport au corps}

Les principales caractéristiques du rapport au corps évoquées par les professionnels étaient la prise en compte de la douleur et le respect de la pudeur et/ou de l'intimité. Certains ont mentionné la composante technique du rapport au corps, le respect du corps et de l'enfant de son image du corps. Le bien-être, l'acceptation du corps et/ou de l'appareillage sont des éléments évoqués pour rendre compte de la composante corporelle de la contention. D'autre part, plus de la moitié des professionnels a évoqué le sens accordé au travail avec et sur le corps de l'enfant dans le service et la conception nécessairement holistique de la personne qui s'y rattache.

Insérer ici le Tableau 1 


\section{Discussion}

Le but de ce travail était d'appréhender la façon dont les représentations sociales de la contention organisent et façonnent le discours des professionnels. Les résultats tendent à montrer que les représentations sociales de la contention vont intervenir dans la régulation de cette pratique, notamment en influant sur le vécu (professionnel - enfant - parent) et la représentation du rôle des parents dans cette pratique.

$\mathrm{Au}$ vu de nos résultats, il apparaît que la contention est un objet polymorphe avec un consensus autour de certaines de ses formes (attaches) mais pas pour d'autres, telles que le maintien de l'enfant ou les barrières de lit. L'absence de consensus nous laisse penser que la représentation de la contention est dans une phase de transformation, au sein de laquelle des éléments anciens cohabitent avec des éléments nouveaux, parfois contradictoires [10]. La difficulté à définir l'objet peut aussi être interprétée au regard de la pluralité des définitions existant dans la littérature scientifique internationale.

Les résultats semblent indiquer que la cause de la contention est multifactorielle et qu'il existe un certain flou sur son aspect systématique ou non. À travers le discours des professionnels, la contention semble notamment être légitimée par l'état des enfants et par l'action préventive de cette pratique. Les croyances au regard des bénéfices de la contention («pour le bien de l'enfant», «pour éviter un danger», etc.) ou des conséquences relatives à l'absence de contention (« risque pour l'enfant», etc.) semblent également jouer un rôle important dans sa mise en place. Ces croyances aident aux décisions et guident les comportements [15]. Ainsi, plus les personnes croient aux bénéfices de la contention, plus celle-ci sera mise en place. En d'autres termes, nous pourrions dire que l'état des enfants et l'action préventive de la contention légitiment la mise en place de cette dernière avant tout en raison des croyances relatives aux bénéfices qu'elle pourrait engendrer par rapport à un risque perçu potentiel qui n'est pas nécessairement réel. Il semble important de former les soignants à la reconnaissance du rôle de leurs croyances afin d'éviter les « abus de contention » [3].

Le fait qu'il n'y ait que peu de prises de positions clairement exprimées pourrait être expliqué du point de vue de la complexité de l'objet et de la difficulté de prendre position relativement à une pratique « ambiguë».

L'absence de consensus sur l'aspect systématique ou non de la contention laisse à penser, une nouvelle fois, qu'il s'agit d'une pratique dont les contours ne sont pas clairement définis pour les professionnels. Il apparaît que celle-ci est « protocolisée » en Réanimation, c'est-à-dire automatique, et qu'elle est mise en place sur prescription médicale dans peu de cas. Cela s'inscrit dans un cadre plus général où plus de la moitié des professionnels semble ignorer 
qu'une contention nécessite une prescription. Cette absence de consensus sur le cadre de la mise en place de la contention (systématique versus ponctuelle) pourrait notamment s'expliquer par l'absence de législation chez l'enfant et par le fait que cette pratique soit peu présente dans la formation des professionnels de santé [2]. De même, différents enjeux, exprimés en particulier par la profession infirmière, soulignent l'existence d'un paradoxe qui a été mis en évidence dans la littérature scientifique. Menier et al. (2010) l'expriment très clairement «sur le plan juridique, la situation des soignants est paradoxale : " un patient pourrait (...) poursuivre un médecin pour lui avoir imposé une contention sans son consentement, mais également pour avoir mis sa santé en danger parce qu'il n'a pas utilisé de moyen de contention (dont on supposera qu'il aurait permis d'éviter une chute) », car «les soignants et l'institution ont une obligation légale et morale de sécurité à l'égard du patient» [16]. D'autre part, cet enjeu relatif à la responsabilité des soignants laisse entendre qu'il existe une « norme subjective » (i.e. la perception d'une pression sociale incitant à réaliser ou à ne pas réaliser un comportement de santé) [17]. À cela s'ajoute le peu de discussion entre les professionnels autour de la décision de la contention, ce qui pourrait également générer une ambiguïté quant à ses conditions de mise en place, bien que la possibilité de communiquer autour de l'objet dans le service soit évoquée par la plupart des professionnels. Enfin, on peut également supposer que la mise en place de la contention sur prescription ou non dépend également du statut qui est accordé à cette pratique. Si la contention est envisagée comme un soin par les professionnels, on peut supposer qu'elle sera alors plus systématiquement mise en place sur prescription médicale au regard de ses bénéfices et de ses risques.

L'importance de la communication avec tous les interlocuteurs concernés est un thème transversal présent à la fois dans les représentations de la contention, en particulier lors de sa mise en place, et dans le cadre plus large de la prise en charge des enfants dans le service. Dans un contexte d'incertitudes, la communication peut avoir pour objectifs de réduire les conséquences de cette situation d'incertitudes. Enfin, la place accordée à la communication pourrait s'expliquer par le fait que la contention est souvent mise en place dans le cadre de soins «problématiques ». Ces derniers activeraient des modalités de pensée contradictoires, générant ainsi un besoin de communication entre professionnels mais également avec les enfants et les parents afin de retrouver un certain équilibre. L'adaptation semble être une composante importante de la pratique de la contention. L'enfant et les parents sont pris en compte dans cette adaptation dans plus de la moitié des cas. En revanche, le fait qu'ils soient peu évoqués par les professionnels tant au niveau des causes, de la décision, que de la mise en place de la contention soulève la question du poids et de la place des enfants et des parents 
dans cette pratique. Ces données questionnent en particulier la place du consentement éclairé dans les soins et, plus largement, de la mise en pratique de la législation relative aux droits des malades, ici des enfants, et des parents. Par ailleurs, le rôle des parents reste difficile à définir pour les professionnels, certains exprimant qu'il est fondamental, d'autres qu'il n'existe pas. Cela amène, plus globalement, à s'interroger la place des parents à l'hôpital. Enfin, la décision de la contention peut également être questionnée au regard de la prise en charge de la douleur.

Thème abordé spontanément par la moitié des professionnels, les alternatives à la contention ne sont cependant pas du tout évoquées par les médecins et les auxiliaires de puéricultures de l'échantillon. Une hypothèse serait que, pour ces professions, il n'existerait pas forcément d'alternatives possibles, que la pratique de la contention serait plus « légitime » ou la réponse la plus adaptée. Les limites de la contention, quant à elles, sont spontanément évoquées par moins de la moitié des professionnels. Faut-il y voir une certaine forme de méconnaissance ou de déni des effets négatifs de la contention? On peut supposer qu'il s'agit d'une forme de rationalisation de l'acte, voire une protection de l'identité professionnelle face à une pratique aux enjeux symboliques et émotionnels importants.

Le vécu de la contention est mitigé, marqué par l'ambivalence du discours, bien que les affects négatifs soient le plus fréquemment évoqués. Ce résultat est cohérent avec la littérature scientifique. Du point de vue des patients par exemple, des auteurs ont montré que la majorité des personnes interrogées percevaient la contention comme étant une intervention «purement négative » et «non thérapeutique »[18]. Les émotions affectent de nombreux processus cognitifs, tels que la perception, l'attention, le raisonnement, la prise de décision ou encore le jugement [19]. Il pourrait donc être intéressant de questionner de manière plus fine la place de ces émotions dans les représentations sociales de la contention, afin de mieux comprendre les processus en jeu. En d'autres termes, le ressenti tient une place prépondérante dans la réaction que les professionnels vont avoir face à la contention et il n'existe pas une seule « réalité » ou «vérité » de la contention. Nous pourrions ainsi dire que le discours des soignants est emprunt d'une certaine forme d'ambivalence. Celle-ci témoigne de la prise de distance avec cette pratique qui, par sa spécificité et les affects qu'elle active, est indissociable d'un contexte évaluatif dans lequel elle prend forme et sens. L'ambivalence pourrait également s'expliquer par le fait que les différents types de contention ne font pas l'objet d'une même évaluation, certaines étant moins perçues négativement que d'autres [20].

Il est également important de noter que la contention chimique n'a été évoquée que par une seule personne au cours de ce travail. Cela nous amène à nous questionner sur la place de 
cette contention dans le service. Les médicaments sont, par ailleurs, peu évoqués par les professionnels, que ce soit dans le cadre de la prise en charge globale de l'enfant ou en tant qu'alternative à la contention. Enfin, il est intéressant de noter que le corps de l'enfant est également très peu évoqué par les professionnels dans le cadre de la contention ce qui nous amène à nous interroger sur la conception que ces derniers ont du corps, porteur de handicap, appareillé, techniqué et à la place accordée à ce corps dans la relation à l'autre et dans le soin. En effet, il peut sembler surprenant que l'objet même sur lequel s'exerce la contention (le corps) soit si peu présent dans le discours des soignants. Faut-il en conclure que le corps est absent de la pratique ? Nous pensons plutôt que son statut d'évidence et son omniprésence le soustraient au travail d'explicitation de la pratique.

Il est intéressant de constater que chacune des personnes interrogées a eu une expérience de l'objet, bien qu'à des degrés divers, y compris les professionnels non soignants (éducatrice, institutrice). Cette pratique ne concernerait donc pas uniquement les soignants mais tous les membres de l'équipe pluridisciplinaire.

La taille de l'échantillon et le fait que notre étude soit mono-centrique peuvent constituer une limite à cette étude. Toutefois, nous avons veillé à avoir un échantillon disposant d'une relative hétérogénéité qualitative. Enfin, le fait que cette recherche se soit déroulée à la suite d'une demi-journée de formation sur la contention au sein de l'Hôpital Raymond Poincaré peut amener à s'interroger sur l'effet de cette intervention sur le discours des participants. Toutefois, il faut garder à l'esprit que les représentations sont résistantes au changement [6], c'est à la suite de modifications des circonstances externes et des pratiques (organisationnelles, pratiques professionnelles) dans le temps que peuvent se mesurer des transformations des représentations préexistantes.

$\mathrm{Au} \mathrm{vu} \mathrm{des} \mathrm{résultats} \mathrm{obtenus} \mathrm{et} \mathrm{des} \mathrm{limites} \mathrm{que} \mathrm{nous} \mathrm{venons} \mathrm{d'évoquer,} \mathrm{il} \mathrm{pourrait} \mathrm{être}$ intéressant d'étudier les spécificités pouvant exister dans les énoncés discursifs des personnes selon leur profession, leur âge et leur ancienneté au sein du service, afin d'appréhender l'effet de ces variables sur les représentations sociales des professionnels. Il semble également important de clarifier le statut et la place de la contention chimique au sein du service car celle-ci est pratiquement absente du discours des professionnels. Approfondir la question du statut de la contention permettrait d'avoir une vision plus globale de cette pratique et de mieux en comprendre les enjeux. Une recherche pourrait également être menée auprès des enfants et des parents afin d'avoir une vue d'ensemble de cette pratique et de ses enjeux pour chacun des acteurs qu'elle implique. Enfin, dans une perspective de compréhension plus large, il pourrait être intéressant de conduire une étude sur la place du corps et le rapport au 
corps dans la relation soignant-soigné dans un contexte de prise en charge de l'enfant lourdement handicapé.

Pour finir, le fait que les connaissances relatives au cadre et à la mise en place de la contention soient variables d'un professionnel à l'autre nous incite à formuler plusieurs préconisations. Il serait, tout d'abord, intéressant de réaliser un état des lieux des connaissances relatives au cadre et à la mise en place de la contention dans le service, en particulier en ce qui concerne la nécessité de la prescription médicale, afin d'avoir une vision globale des représentations sociales et des connaissances qui sont acquises ou qui restent à approfondir. À partir de cet état des lieux, des modules de formation pourraient être élaborés afin de répondre aux besoins qui auront été dégagés. D'autre part, des groupes d'échanges de pratique permettraient d'inscrire la contention dans une logique de réflexion commune et de clarifier son cadre et ses modalités, afin de tendre vers un niveau de connaissance uniforme de l'objet. Enfin, communiquer autour de la contention, que ce soit au sein de l'équipe pluridisciplinaire, avec les enfants hospitalisés ou avec leur famille, permettrait de formaliser davantage cette pratique et de lui redonner du sens en mettant en évidence son intérêt dans les cas où elle s'avère nécessaire.

\section{Conclusion}

À travers ce travail, nous souhaitions comprendre comment les représentations sociales des professionnels intervenaient dans la pratique de la contention dans un contexte de soins et de prise en charge de l'enfant malade et/ou handicapé. Les résultats émergeants de l'analyse de contenu nous laissent à penser que les représentations sociales jouent en particulier sur le vécu des professionnels et la représentation qu'ils peuvent avoir du vécu des enfants, des parents et du rôle de ces derniers dans la contention. Les résultats nous montrent également que la contention est un objet qui pose question et suscite une grande ambivalence dans le discours des professionnels, que ce soit à travers ses formes ou le vécu relatif à cette pratique. On peut ainsi observer un véritable travail de légitimation et/ou de délégitimation de la pratique, nuancé par cette ambivalence du discours et les contours flous de l'objet. Par ailleurs, la place et le rôle de chacun au cœur de cette pratique semblent difficiles à définir par les professionnels, tout comme le cadre dans lequel elle s'inscrit. Cela nous conduit à envisager la contention comme un objet pluridimensionnel en transformation, indissociable de son inscription dans un contexte de questionnement actuel et autour duquel s'articulent différents enjeux. Ceux-ci concernent aussi bien les différents acteurs impliqués dans la contention (enfant - professionnels - parents) que les politiques de santé actuelles. Cette recherche a 
ainsi fait émerger divers questionnements et éléments de réflexion qui peuvent représenter de véritables leviers pour parvenir à une meilleure compréhension de la mise en œuvre de la contention, des enjeux pour les différents acteurs impliqués et pour dresser les lignes directrices d'une réflexion sur la formation des soignants.

\section{Déclaration d'intérêts}

Les auteurs déclarent ne pas avoir de conflits d'intérêts en relation avec cet article.

\section{Références}

[1] Annequin D. Contention pour les gestes douloureux, les risques de maltraitance. Pédiatrie Pratique 2011;228:4-5.

[2] Agence Nationale d'Accréditation et d'Évaluation en Santé. Évaluation des pratiques professionnelles dans les établissements de santé: Limiter les risques de la contention physique de la personne âgée 2000;1-42.

[3] Kinoo P, Kpadonou-Fiossi E. Enfant, contenance et contention. Neuropsychiatrie de l'enfance et de l'adolescence 2008;56:117-121.

[4] Williams D. Restraint Safety: an Analysis of Injuries Related to Restraint of People with Intellectual Disabilities. Journal of Applied Research in Intellectual Disabilities 2009;22:135139.

[5] Braitman A, Guedj MJ. Mesures de contention sans isolement. Revue de la littérature et des recommandations en vigueur. Journal de Nervure 2003;16:1-5.

[6] Abric JC. Pratiques sociales et représentations. Paris: PUF; 1994.

[7] Doise W. L'explication en psychologie sociale. Paris: PUF; 1982.

[8] Boutry L, Matheron I, Bidat E. (2001). Quand les prescriptions ne sont pas suivies... penser aux croyances et représentations de santé. L'exemple du patient asthmatique. Revue Française d'Allergologie et d'Immunologie Clinique 2001;41:470-476.

[9] Salas S, Dany L, Michel R, Cannone P, Dudoit E, Duffaud F, Favre R. Représentations, attitudes et pratiques associées aux soins palliatifs : une enquête auprès des responsables d'unités et d'équipes de soins palliatifs en France. Médecine Palliative 2007;7: 130-139.

[10] Jodelet D. Les représentations sociales. Paris: PUF; 1989.

[11] Dany L, Dudoit E, Salas S, Duffaud, F. Les représentations des soins palliatifs. In: SaintJean M, Bastiani F. editors. L'épreuve de la fin de vie. Paris: Seli Arslan; 2014.

[12] Moscovici S, Buschini F. Les méthodes des sciences humaines. Paris: PUF; 2003.

[13] Bardin L. L'analyse de contenu. Paris: PUF; 2007.

[14] Negura L. L'analyse de contenu dans l'étude des représentations sociales. SociologieS 2006. Disponible sur internet : URL: http://sociologies.revues.org/index993.html. Consulté le 01/09/2012. 
[15] Cestac J, Meyer T. Des attitudes à la prédiction du comportement: le modèle du comportement planifié. In: Morchain P, Somat A. editors. La psychologie sociale: applicabilité et applications. Rennes: PUR; 2010.

[16] Menier C, Rodriguez $\mathrm{B}$, Lassaunière $\mathrm{JM}$, et al. La contention physique passive: une enquête de prévalence dans un centre hospitalo-universitaire. Médecine palliative - Soins de support - Accompagnement - Éthique 2010;9:232-241.

[17] Morin M. Parcours de santé. Paris: Armand Colin; 2004.

[18] Chien WT, Chan C, Lam LW, et al. Psychiatric inpatients' perceptions of positive and negative aspects of physical restraint. Patient Education and Counseling 2005;59:80-86.

[19] Niedenthal PM, Krauth-Gruber S, Ric F. Comprendre les émotions: Perspectives cognitives et psycho-sociales. Bruxelles: Mardaga; 2009.

[20] Cunningham J, McDonnell A, Easton S, et al. Social validation data on three methods of physical restraint: views of consumers, staff and students. Research in Developmental Disabilities 2003;24:307-316. 


\section{Annexes}

\section{Guide d'entretien}

- Pouvez-vous me parler de votre travail auprès des enfants du service ?

- Qu'est-ce qui fait la spécificité du travail dans ce service selon vous?

- Y a-t-il des choses plus difficiles pour vous dans le service?

- Avez-vous rencontré des difficultés en particulier?

- Pour vous, que signifie travailler sur/avec le corps des enfants ?

- Que vous évoque cette question?

- Est-ce que vous différenciez les enfants du service ? (si oui, comment ? si non, pourquoi ?)

- Comment s'effectue le travail sur le corps de l'enfant dans le service ?

- Pour vous, quels sont les enjeux associés au travail sur l'enfant et son corps ?

- Qu'est-ce que le terme contention évoque pour vous?

- Est-ce que vous pouvez m'expliquer comment on décide de mettre en place une contention?

- Selon vous, comment la contention devrait-elle être mise en place?

- Est-ce que vous avez déjà utilisé une contention auprès d'un enfant ?

— Si oui : Qu'est-ce que vous en avez pensé ? Qu'est-ce que ça vous a évoqué ? Qu'avez vous ressenti à cette occasion ? Est-ce que vous avez la possibilité d'en discuter après ?

- Si non : Est-ce que vous avez déjà vu quelqu'un poser une contention sur un enfant? Qu'est-ce que vous en avez pensé ? Qu'est-ce que ça vous a évoqué ? Qu'avez -vous ressenti à cette occasion ? Est-ce que vous avez la possibilité d'en discuter après?

- Pourriez-vous me parler du rôle des parents dans la contention?

- Pouvez-vous me raconter la dernière contention que vous avez vue ou effectuée? 